\title{
The transmission pattern and clinical course identified from 417 patients with COVID-19 pneumonia in Shenzhen, China: a retrospective and observational study
}

\section{Feijuan Huang}

Shenzhen University First Affiliated Hospital: Shenzhen Second People's Hospital

\section{Yu Zhang}

Shenzhen University First Affiliated Hospital: Shenzhen Second People's Hospital https://orcid.org/0000-0003-0285-239X

\section{Yuanzhe Cai}

Shenzhen Technology University

\section{Feng Ding}

Shenzhen University First Affiliated Hospital: Shenzhen Second People's Hospital

\section{Zhanyan Liu}

Shenzhen University First Affiliated Hospital: Shenzhen Second People's Hospital

Jieren Liu

Shenzhen University First Affiliated Hospital: Shenzhen Second People's Hospital

\section{Yan Liu}

Shenzhen University First Affiliated Hospital: Shenzhen Second People's Hospital

\section{Yaowei Huang}

Shenzhen University First Affiliated Hospital: Shenzhen Second People's Hospital

\section{Xiaomiao Tian}

Shenzhen University First Affiliated Hospital: Shenzhen Second People's Hospital

\section{Weldon Lee}

Houston Methodist Research Institute

\section{Zhengzhi Wu}

Shenzhen University First Affiliated Hospital: Shenzhen Second People's Hospital

Shiwei Yang ( $D$ 1063993905@qq.com )

Shenzhen University First Affiliated Hospital 3002 Sungang West Road https://orcid.org/0000-00019570-1800

\section{Research}


Keywords: COVID-19, clustered cases, sporadic cases, pandemic, public health care

Posted Date: June 9th, 2021

DOI: https://doi.org/10.21203/rs.3.rs-596908/v1

License: (c) (1) This work is licensed under a Creative Commons Attribution 4.0 International License. Read Full License 


\section{Abstract}

\section{Background}

To help government formulating epidemic control strategies and spreading Shenzhen's experience.

\section{Methods}

417 patients admitted to hospital with confirmed COVID-19 infection between 19 January and 21 February 2020 in Shenzhen, China.Observational study of COVID-19 outcomes using quality-assured public data from website of Shenzhen Municipal Health Commission and a COVID-19 diagnosis and treatment fixed-point hospital to transmission patterns and associated factors of sporadic and clustered COVID-19 cases and its critical time courses.

\section{Results}

We Compared the characteristics of clustered and non-clustered cases, found the clustered cases differed significantly from non-clustered cases in age and exposure history. Moreover, we analyzed the time intervals between symptom onset and recovery for three clinical conditions and different stages of transmission patterns, found the time intervals between illness onset and hospital discharge for all patients were no more than 30 days. Finally, we analyzed disease severity conditions, severe patients spent 4-5 more days of hospitalization and medical intervention than moderate and mild patients did.

\section{Conclusions}

The study confirms that severe patients spent 4-5 more days of hospitalization and medical intervention than moderate and mild patients did. Our results also provided robust evidence that timely and effective prevention measures were the key to quickly cut the transmission chains and prevent the transition from clustered transmission pattern to community transmission pattern.

\section{Background}

Coronavirus disease 2019 (COVID-19) is a type of pneumonia due to infection with SARS-CoV-2. ${ }^{1-5}$ Cluster transmission is an important transmission route of COVID-19.6,7 Clustered cases are defined as two or more cases occurring in one family or within the same region. The population transmission pattern and the pathogenetic characteristics are of significant importance in preventing the outbreak of COVID$19 . .^{8-11}$

As a heavily populated city with the largest floating population in China (average daily floating population: above 25 million), Shenzhen is the city where the first COVID-19 case was imported and diagnosed in China except Wuhan. As the spreading of COVID-19 outbreak from Wuhan to other cities in China, Shenzhen ranked among the top cities outside Wuhan with a reported 417 confirmed cases by February 21, 2020. Among all these laboratory-confirmed COVID-19 cases in Shenzhen between January 
19,2020 and April 30, 2020, there were only 3 death cases with a mortality rate of $0.72 \%$. The prevention of COVID-19 infection was quite a success.

At present, information regarding the transmission pattern and the clinical course of pneumonia caused by $2019-n C o V$ is scarce. ${ }^{6,12}$ Analyses of patients are particularly useful to infer key epidemiological parameters, such as the incubation between exposure and illness onset, self-observation periods between illness onset and medical visit, diagnosis periods between the medical visit and the reporting of cases, treatment periods between the reporting date and hospital discharge or death.

In this study, we did a comprehensive exploration of the transmission pattern and epidemiological time course of 417 patients with confirmed 2019-nCoV pneumonia in Shenzhen by February 21, 2020. Our data shows that the epidemic of 2019-nCoV pneumonia in Shenzhen has epidemic characteristics such as rapid rise in epidemics and short duration. However, the incidence of the disease decreased rapidly, which shows that the effect of the intervention is practical. Therefore, early detection of cases and early isolation are useful measures to reduce morbidity. These characteristics can be used as a theoretical basis for formulating epidemic control strategies, improving prevention and control efficiency, and reducing the waste of medical and health resources. With the spread of the epidemic in the world, under the background of globalization and the concept of building a community of shared future for humankind, Shenzhen's experience has reference value.

\section{Methods}

\section{Case collection}

A total of 417 patients enrolled in this study were diagnosed with COVID-19 pneumonia in Shenzhen between January 192020 and February 21, 2020 according to the Chinese Center for Disease Control and Prevention (CCDC) interim guidance. ${ }^{13}$ These patients showed typical clinical phenotype of COVID-19 pneumonia and were laboratory-confirmed using real-time fluorescent RT-PCR. Characteristics of patients were collected from the official website of the Health Commission of Shenzhen Municipality, including age, sex, occupation, residential district, date of illness onset, date of a first medical visit, date of hospital discharge, date of death, exposure history, the severity of illness and relationship with other patients as listed in Table 1. All the data of included cases have been shared with WHO.

\section{Table 1 Characteristics of population distribution in clustered cases versus non-clustered cases}




\begin{tabular}{|c|c|c|c|c|c|}
\hline \multirow[t]{2}{*}{ Basic characteristics } & \multirow[t]{2}{*}{$\begin{array}{l}\text { Subtotal } \\
(n=417)\end{array}$} & Clustered & $\begin{array}{l}\text { Non- } \\
\text { clustered }\end{array}$ & $x^{2}$ & $P$ value \\
\hline & & $\begin{array}{l}\text { cases } \\
(\mathrm{n}=235)\end{array}$ & $\begin{array}{l}\text { Cases } \\
(n=182)\end{array}$ & & \\
\hline Median age (years) & $47.00(1-86)$ & $48.00(2-86)$ & $47.00(1-81)$ & & \\
\hline Average age $\pm S D$ & $45.38 \pm 17.68$ & $44.76 \pm 19.42$ & $46.19 \pm 15.17$ & & \\
\hline \multicolumn{6}{|l|}{ Age (years) distribution n (\%) } \\
\hline$<=10$ & $23(5.52 \%)$ & $22(9.36 \%)$ & $1(0.55 \%)$ & 13.857 & 0.0002 \\
\hline $11-20$ & $16(3.84 \%)$ & $9(3.83 \%)$ & $7(3.85 \%)$ & 0 & 1 \\
\hline $21-30$ & $36(8.63 \%)$ & $14(5.96 \%)$ & $22(12.09 \%)$ & 4.084 & 0.0433 \\
\hline $31-40$ & $93(22.30 \%)$ & $51(21.70 \%)$ & $42(23.08 \%)$ & 0.071 & 0.7899 \\
\hline $41-50$ & $66(15.83 \%)$ & $37(15.74 \%)$ & $29(15.93 \%)$ & 0.002 & 0.9643 \\
\hline $51-60$ & $82(19.66 \%)$ & $38(16.17 \%)$ & $44(24.18 \%)$ & 2.773 & 0.0959 \\
\hline $61-70$ & $85(20.38 \%)$ & $55(23.40 \%)$ & $30(16.48 \%)$ & 2.018 & 0.1554 \\
\hline $71-80$ & $14(3.36 \%)$ & $8(3.40 \%)$ & $6(3.30 \%)$ & 0.003 & 0.9563 \\
\hline$>80$ & $2(0.48 \%)$ & $1(0.43 \%)$ & $1(0.55 \%)$ & 0.033 & 0.85589 \\
\hline total & 417 & 235 & 182 & 24.784 & 0.0017 \\
\hline \multicolumn{6}{|l|}{ Sex } \\
\hline Male & 198(47.48) & $106(45.11 \%)$ & $92(50.55 \%)$ & 0.432 & 0.511 \\
\hline Female & $219(51.52)$ & $129(54.89 \%)$ & $90 \rrbracket 49.45 \% \rrbracket$ & 0.381 & 0.5371 \\
\hline total & 417 & 235 & 182 & 1.219 & 0.2696 \\
\hline \multicolumn{6}{|l|}{ Origin-of-case distribution (\%) } \\
\hline $\begin{array}{l}\text { Coming to Shenzhen from } \\
\text { Wuhan }\end{array}$ & $107(25.66 \%)$ & $45(19.15 \%)$ & $62(34.07 \%)$ & 6.997 & 0.0082 \\
\hline $\begin{array}{l}\text { People coming to Shenzhen } \\
\text { from Hubei other than Wuhan }\end{array}$ & $33(7.91 \%)$ & $18(7.66 \%)$ & $15(8.24 \%)$ & 0.041 & 0.8395 \\
\hline Local Shenzhen residents & $260(62.35 \%)$ & $165(70.21 \%)$ & $95(52.20 \%)$ & 3.346 & 0.0674 \\
\hline Others & $17(4.08 \%)$ & $7(2.98 \%)$ & $10(5.49 \%)$ & 1.526 & 0.2167 \\
\hline Total & 417 & 235 & 182 & 15.869 & 0.0012 \\
\hline \multicolumn{6}{|l|}{ Exposure history n (\%) } \\
\hline Wuhan contact history & $220(52.76 \%)$ & 103(43.83\%) & $117(64.29 \%)$ & 5.259 & 0.0218 \\
\hline
\end{tabular}




\begin{tabular}{|lcllll|}
$\begin{array}{l}\text { Hubei contact history other } \\
\text { than Wuhan }\end{array}$ & $87(20.86 \%)$ & $57(24.26 \%)$ & $30(16.48 \%)$ & 2.48 & 0.1153 \\
$\begin{array}{l}\text { Travel to other cities other than } \\
\text { Hubei }\end{array}$ & $25(6.00 \%)$ & $17(7.23 \%)$ & $8(4.40 \%)$ & 1.305 & 0.2533 \\
$\begin{array}{l}\text { Shenzhen residents with no } \\
\text { travel history }\end{array}$ & $39(9.35 \%)$ & $30(12.77 \%)$ & $9(4.95 \%)$ & 6.198 & 0.0128 \\
\hline $\begin{array}{l}\text { Contact with confirmed } \\
\text { patients }\end{array}$ & $20(4.80 \%)$ & $15(6.38 \%)$ & $5(2.75 \%)$ & 2.71 & 0.0997 \\
\hline Others & $26(6.24 \%)$ & $13(5.53 \%)$ & $13(7.14 \%)$ & 0.401 & 0.5266 \\
\hline Total & 417 & 235 & 182 & 22.444 & 0.0004 \\
\hline Disease severity $\mathbf{n ( \% )}$ & $28(6.71 \%)$ & $12(5.11 \%)$ & $16(8.79 \%)$ & 0.571 & 0.4496 \\
\hline Mild & $376(90.17 \%)$ & $213(90.64 \%)$ & $163(89.56 \%)$ & 6.649 & 0.0099 \\
\hline Moderate & $12(0.48 \%)$ & $9(3.83 \%)$ & $3(1.65 \%)$ & 0.256 & 0.6129 \\
\hline Severe & $1(0.24 \%)$ & $1(0.43 \%)$ & $0(0.00 \%)$ & 1.0 & 0.3173 \\
\hline Others & 417 & 235 & 182 & 5.235 & 0.3878 \\
\hline Total & & & & & \\
\hline
\end{tabular}

Note: Each non-clustered case residing in both Shenzhen and Wuhan is deemed as 1 person coming to Shenzhen from Wuhan

\section{Cases definitions}

Clustered COVID-19 cases were defined as 2 or more confirmed cases with contact history in a defined small area, such as an apartment or house, a working organization, a community, etc. The first case in each cluster was defined as the case with the earliest date of illness onset, but not the earliest date of diagnosis. The onset date was defined as the date when the patient complained any COVID-19-related symptoms at a medical visit, and the confirmation date was defined as the date on which laboratory confirmation was obtained for SARS-CoV-2 infection.

The rest cases in a cluster group were required to fully comply with the following three rules: 1) there was a history of contact with the first case within 14 days before the onset of symptoms; 2) there was no history of travel to Wuhan and surrounding areas or to other communities with reported cases; 3 ) there was no other history of Co-exposure cases in each cluster of outbreaks were defined as patients who had a common exposure history with the first case and could not distinguish who was the first case.

According to the relationship among the cases in a clustered epidemic group, the clustered groups were divided into family gathering, party gathering or dinner gathering, travel gathering, working gathering, hospital gathering, other close contact gatherings, and there were mixed gatherings consisting of several gathering scenarios. 
Based on the contact history all cases were classified as follows: (1) Wuhan/Hubei residents who came to Shenzhen from Wuhan/Hubei 14 days prior to illness onset; (2) with a travel history to Wuhan/Hubei 14 days prior to illness onset; (3) with contact history with Wuhan/Hubei residents 14 days prior to illness onset; (4) with no travel history to Wuhan/Hubei or no contact history with Wuhan/Hubei residents 14 days prior to illness onset.

\section{Statistical analysis}

All data were analyzed with descriptive statistics, comparability analysis between clustered cases and non-clustered cases of COVID-19 were carried out by $X^{2}$ test in terms of date of onset, clan distribution, sex, age, source-of-infection exposure history, peak characteristics, and incubation period.

\section{Patient and public involvement}

Patients and/or the public were not involved in the design, or conduct, or reporting, or dissemination plans of this research.

\section{Results}

\section{Comparison of clustered cases versus non-clustered cases}

Basic characteristics of patient distribution in clustered cases versus non-clustered cases were shown in Table 1. By February 21, 2020, there were a total of 417 confirmed cases, of which imported cases were predominant: $84.41 \%$ of the cases had Hubei/Wuhan exposure history; and most of them $(96.88 \%)$ were in mild and stable conditions. As showed in table 1, the ages of illness onset ranged from 1 year to 86 years, the average age was $45.38 \pm 17.68$ years, the median age was 47 years, and the male-to-female ratio was 1:1.1.

As listed in Table 1, in the clustered cases, the median age was 48 years and the average age was $44.76 \pm$ 19.42 years. The average age in the clustered cases was a little younger than that in the non-clustered cases. In the age group of 1 10 years, the number of the clustered cases was much more than that of the non-clustered cases $\left(X^{2}=13.857, P\right.$ value $\left.=0.0002\right)$. In the age group of $>60$ years, the number of the clustered cases was also more than that of the non-clustered cases, but the difference did not reach statistical significance $\left(X^{2}=1.638, P\right.$ value $\left.=0.2006\right)$. However, in the other two age groups, i.e., $21-30$ years and 51-60 years, the numbers of the clustered cases were less than that of non-clustered cases, with a statistical results of $X^{2}=4.084, P$ value $=0.0433$ ) and $X^{2}=2.773, P$ value $=0.0959$, respectively. These data indicated that the clustered cases differed significantly from non-clustered cases in age group distribution, with a significantly higher percentages of clustered cases in the age group of 1-10 years than that of non-clustered cases $(9.36 \%$ versus $0.55 \%)$ and a significantly lower percentage of clustered cases in the age group of $21-30$ years than that of non-clustered cases (5.96\% versus $12.09 \%$ ). 
Between clustered and non-clustered cases, there were differences in terms of disease severity, the percentage of clustered cases with severe and critical conditions appeared more than that of nonclustered cases with same conditions $\left(3.83 \%\right.$ versus $1.65 \%, X^{2}=0.256, P$ value $\left.=0.6129\right)$. In the clustered group, 160 cases were having Hubei/Wuhan exposure history (68.09\%), and in the non-clustered group, 147 cases were having Hubei/Wuhan contact history (80.77\%), but this difference was not statistically significant $\left(X^{2}=1.281, P\right.$ value $\left.=0.2577\right)$. To our surprise, the percentage of patients with a Hubei/Wuhan exposure history was markedly lower in the clustered group than that in the non-clustered group $(P<$ $0.001)$.

\section{Comparison of time course of the clustered cases versus non-clustered cases}

To better understand the transmission patterns for the clustered and non-clustered cases, we made a follow-up study on the time course of the disease progression, including time points of exposure, illness onset, medical visit, diagnosis and hospital discharge/death. The comparisons for date of exposure (Figure 1 A), illness onset (Figure 1 B), first medical visit (Figure 1 C), cases confirmed (Figure 1 D), hospital discharge/death (Figure $1 \mathrm{D}$ ) between the clustered cases and the non-clustered cases were shown in Figures 1. The first COVID-19 patient in Shenzhen was admitted on January 9, 2020区Figure 1C with the reported illness onset date of January 1, 2020 (Figure 1A) and exposure date of December 26, 2019 (Figure 1C) in Wuhan. The number of confirmed cases of COVID-19 began to be released daily on January 19, 2020 (Figure 1D).

Among the domestic 417 cases including 235 clustered and 182 non-clustered cases, there were 110 traceable sporadic cases (accounting for $60.44 \%$ in 182 sporadic cases) and 135 traceable clusteredcases (accounting for $57.45 \%$ in 235 clustered-cases). We compared their exposure time and found that most of them were exposed before January 25, especially between January 10-25 (Figure 1A). We then compared the time of illness onset for 181 sporadic cases (99.45\%) and 225 clustered cases $\nabla 95.74 \% \nabla$ and the data showed that the dates of illness onset for both sporadic and clustered cases were mainly between January 18 and February 6 (Figure 1B), and in which the illness onset time peak of the sporadic group (January 22) was 1-2 days earlier than that of the clustered group (January 24).

Probably due to timely intervention, sporadic cases did not spread to cause more infections, so the peak time of the medical visit of sporadic cases (Jan 22-24) was earlier than that of the clustered cases (Jan 29-31), as shown in Figure 1C). As a result, the overall discharge peak time of sporadic cases (Feb 11) was earlier than that of the clustered cases (Feb 16-21) as shown in Figure 1E, in which 143 clustered cases and 122 non-clustered cases with clear discharge time record were enrolled in the analysis. Due to the rapid intervention response, the secondary infected cases were quickly diagnosed using nucleic acid testing and isolated after the diagnosis of the first case in the clustered cases. From the statistical data, the peak diagnosis dates of scattered cases and clustered cases were almost identical, and the majority cases in each group were diagnosed from January 29 to February 6 (Figure 1D).

\section{Clustered cases and Typical clustered-case groups}


This retrospective analysis showed that the symptom onset date of the first case in Shenzhen was January $1^{\text {st }}, 2019$, which resulted in 5 clustered cases through family transmission (they were tested positive with COVID-19 after January $19^{\text {th }}$.). On January $19^{\text {th }}$, the National Health Commission of China officially confirmed the first imported COVID-19 case in Shenzhen, but in fact, before that time, there were already 10 groups of clustered cases. Then 27 groups appeared in the following week 3 groups ( 7 cases) of which occurred on January 18, then followed by 3 groups ( 8 cases) on January 19, 5 groups (18 cases) on Jan. 20,7 groups (25 cases) on Jan. 23, and 9 groups (30 cases) on January 24 , as shown in Figure 2A. Since the lockdown of Wuhan city on January 23, China has evolved into a critical phase of national epidemic prevention, and the illustrated clustered cases became well contained by home quarantine. After February 10, the origins of the clustered cases were basically under control.

There was a total of 417 diagnosed COVID-19 cases in Shenzhen, of which 235 clustered cases consisted of 86 clustered groups. Among them, 160 cases (69 clustered group) had Hubei/Wuhan exposure history. There were 86 clustered groups, 1 group with 9 clustered cases, 2 groups with 6 clustered cases each, 4 groups with 5 clustered cases each, 8 groups with 4 clustered cases each, 20 groups with 3 clustered cases each, and 51 groups with 2 clustered cases each. Notably, the majority of the clustered groups had 2 or 3 members, which accounted for more than $70 \%$ of the cases.

Among the 235 clustered cases, 5 cases were due to colleagues transmission, 3 cases were due to friends transmission, 227 cases were due to family transmission, and thus more than $90 \%$ of the clustered cases were derived from family clustering (Figure 2B).

\section{Comparison of the first case and the secondary cases in clustered groups}

In order to study the transmission characteristics of clustered cases, we obtained the detailed transmission route for each clustered group based on the time of illness onset, medical visit, case confirmation and hospital discharge/death, and this analysis would allow an in-depth comparison of the transmission characteristics for the first and secondary cases in each clustered group.

As shown in Figure 3A, the median time interval of illness onset between the first and secondary cases was 3 days and the average time intervals of illness onset were $4 \pm 4$ days. The median time interval of the first medical visit between the first and secondary cases was 1 day and the average time interval of illness onset was $2.4 \pm 4$ days (Figure 3B). The median time interval of confirmation time between the first and secondary cases was 1 day and the average time interval of illness onset was $1.5 \pm 3.4$ days (Figure 3C). The median time interval of hospital discharge/death between the first and secondary cases was -1 days and the average time interval of illness onset was $-0.6 \pm 7$ days (Figure 3D).

\section{COVID-19 epidemic control in Shenzhen}

As shown in Figure 4A, the time intervals between illness onset and hospital discharge for the patients in severe ( 26 days) and moderate ( 25 days) conditions were almost the same. As expected, due to mild symptoms, the interval between onset and discharge of patients with mild illness is relatively short (22 
days). The median time intervals between medical visits and diagnosis for severe, mediate, and mild patients were 2-3 days (Figure 4B and 4C), indicating that it took almost the same time for most patients to get diagnosed from their first medical visit. As shown in Figure 4B, the median time intervals between a hospital visit and hospital discharge, i.e., treatment period, for patients in severe, moderate, and mild conditions were 19 days, 17 days, and 18 days, respectively. Apparently, the treatment time for most patients is roughly the same, regardless of their condition.

Due to the relatively severe clinical condition of critically ill patients, the time interval between symptom onset and a hospital visit was relatively short, with the median time interval of 3 days (Figure 4A and 4B). Due to the relatively stable conditions of moderate and mild patients, the time interval between illness onset and medical intervention was relatively long, with median time intervals of 9 days and 4 days.

As shown in Figure 4C, the median time intervals between diagnosis and discharge for severe, moderate, and mild patients were 19 days, 15 days, and 15 days, respectively. The average number of days between diagnosis and discharge for severe, moderate, respectively, indicating that severe patients needed 4-5 more days of medical intervention than moderate and mild patients.

We also analyzed the rates of severe complications including severe pneumonia and death. As shown in Figure 4D, CoVID-19 infection occurred more frequently among old people and could also more likely result in severe and even fatal respiratory diseases such as acute respiratory distress syndrome (ARDS) in old people than young people these people. Respiratory infection symptoms occurred in the early stages of this pneumonia. The majority of the, patients (80\%) exhibited mild or median symptoms and showed good prognosis, while $20 \%$ of the patients developed ARDS, acute respiratory failure and other serious complications. Among the 417 2019-nCoV cases diagnosed in Shenzhen, 48 severe patients developed severe pneumonia, pulmonary edema and ARDS, including that 3 patients who developed multiple organ failure and died.Dates of the percentage of severe patients in Figure 4F. Early identification and timely treatment of critical cases of 2019-nCoV were of great significance in reducing the severity rate of patients (Figure 4E) and effective life support and active treatment of complications could effectively reduce the fatality rate.

Timely implementation of various control measures effectively prevented cross infection and epidemic transmission in Shenzhen. The first COVID-19 case was confirmed on January 19, 2020, and the epidemic situation was under control on March 9, 2020 ( 0 new COVID-19 case within 24 h). During this 50-day period, no health-care workers were infected. As of March 9, 2020, there were only 3 deaths caused by COVID-19 (with a mortality of $0.72 \%$ ), demonstrating that various epidemic prevention measures in Shenzhen had achieved a great outcome. After the implementation of all these strategies and measures, the COVID-19 cases started to decline since February 6 . There were almost no community transmission and nosocomial infection occurred in Shenzhen. Main preventive policies and strategies of COVID-19 in Shenzhen, which could be found on the website of the National Health Commission of China (http://www.nhc.gov.cn/), Health Commission of Guangdong Province (http://wsjkw.gd.gov.cn/), and Shenzhen Municipal Health Commission (http://wjw.sz.gov.cn/). 


\section{Discussion}

After the occurrence of this COVID-19 epidemic in Wuhan, Shenzhen municipal government and related agencies responded quickly and implemented a number of public epidemic prevention measures in time. On January19, 2020, the first COVID-19 patient was diagnosed in Shenzhen. As early as December 31, 2019, ${ }^{14-16}$ Shenzhen Disease Control Center launched an epidemic control program, and its epidemic response strategy has been fully recognized and praised by WHO. ${ }^{17} \mathrm{~A}$ series of early preventive strategies were implemented, which included detection of body temperature at all entrances of main traffic and buildings, outpatients service especially for patients with fever in all main hospitals in Shenzhen. All the patients with fever were screened with nasal or throat swab PCR detection of coronavirus 2019, Chest CT and blood lymphocyte counting in order to find out the early case of COVID-19. Medical observation was established in every public hospital and a designated hospital (Shenzhen $3^{\text {rd }}$ People's Hospital) was responsible for admission and medical care of all confirmed cases. Protection procedure was established for all medical staff who carry on the screening and take care of suspected and confirmed cases. Fourteen days of isolated observation of all subjects arrived at Shenzhen with travel history had been implemented since February 2.

In this study, clinical epidemiological patterns and featured clinical time course of the overall cases were systematically analyzed. We found that the clustered cases were mainly due to familial clustering, and the subsequent patients had close contact with the previous generation cases in the symptomatic phase. Therefore, preventing clustered cases from rapid growing helps reduce the general severity of the disease. In regard to COVID-19 prevention and quarantine, concerns must be paid to home quarantine and inhouse quarantine, which is effective in severe disease prevention. In addition, the clustered cases and the non-clustered cases had no significant difference in the incubation periods.As shown in Figure 5.

It only took 50 days for Shenzhen to control the epidemic situation (no new domestic case) from diagnosis of the first case; no infection of health-care workers reported throughout this period; and as of March 9 , there were only 3 death cases, with a mortality of $0.72 \%$. It is worthwhile for other cities and areas to learn the public health-care prevention strategy of Shenzhen. Early identification and timely treatment of the patients of CoVID-19 are of great importance in not only preventing the spread of this new coronavirus in China and worldwide but also in reducing the death rate.

The mortality of SARS-CoV has been reported as more than 10\%, MERS-CoV at more than $35 \%$ and SARSnCoV-2 at more than $10 \%$ in previous study. ${ }^{15,18,19}$ At data cutoff for this study, the mortality of the 417 included patients infected by CoVID-19 in Shenzhen was $0.72 \%$. The timing of illness onset of severe patients was of great importance, and timely transfer, isolation and effectively supporting treatment are also very critical in reducing mortality. Since January 11,2020 , all suspected cases have been tracked and immediately enrolled in medical observation in designated places (mainly in hotels equipped with health-care workers). All costs of CoVID-19 treatment of Chinese citizens are covered by medical insurance in China. Shenzhen has experienced SARS outbreak, thus has experience in the treatment of respiratory distress syndrome, which is helpful in dealing with the COVID-19 epidemic. 
Although MERS-CoV and SARS-CoV have also been found to infect more males than females, ${ }^{20,21}$ we observed an increased susceptibility of woman than man (females: males=1.1:1) in the 417 cases of 2019-nCoV infection. The three unfortunate death cases were males in the 417 cases of 2019-nCoV infection. The reduced mortality of females could be attributed to the protection from sex hormones that play an important role in innate and adaptive immunity. ${ }^{22}$

The reason for this low mortality rate is mainly attributed to early treatment of severe patients, timely identification of patients with aggravated conditions, and early intervention treatment consisting of screening and diagnosis performed by experts in critical care medicine, respiratory medicine, infection department and imaging subjects. The team conducts screening, evaluation, timely adjustment of diagnosis and treatment measures and correction of potentially reversible factors that aggravate the condition of the patients at least once a day, and strives to alleviate the condition to avoid aggravation and death.

For the key time events of exposure, morbidity, medical visit, diagnosis and hospital discharge of the infection cases in Shenzhen, we carried out a systematically statistical analysis. In the clustered-infection groups, the exposure interval between the first case and the secondary infection cases was 3 days, the onset interval was 3 days, the visit interval was 1 day, the diagnosis interval was 1 day, and the discharge interval was -1 day, which indicates an immediate tracking for the suspected cases.

Compared with the time intervals between infection and recovery in the patients with severe, moderate and mild conditions, shortened clinical duration was observed for mild or moderate cases, implicating that prolonged time course of viral elimination was associated with poor prognosis. Delayed virus diagnosis and delayed patient isolation were associated with prolonged virus elimination duration. Besides, effective patient isolation and timely medical treatment obviously improve the outcome of COVID-19 patients. In general, emphasizing that fast diagnosis and timely patient isolation were effective measures to improve the prognosis of the patients.

To sum up our statistical analysis on the perspective and observative of COVID-19 disease in Shenzhen, we analyzed the whole infection and recovery courses for different patients' clinical condition (severe, moderate, mild), and for different stages of transmission pattern (sporadic, clustered, or community transmission). As the results shown here, there is no significant difference in the time interval between infection and recovery (the time interval between exposure and discharge), regardless of the different transmission patterns. For new coronavirus pneumonia, the time interval between infection and recovery was 30 days, which was a constant for a defined immune system in patients under the circumstance of no effective cure methods existing now.

Timely and effective prevention measures can quickly cut the transmission chains from the development of clustering pattern to community transmission, and return clustered transmission status to sporadic transmission status until the new cases disappear. This reversal of the state from clustered transmission 
to the state of sporadic transmission can significantly reduce the infection rate and the severe patient's rate, thereby reducing mortality.

There are still limitations in this study, firstly on the time course calculation, most of the severe patients that we have tracked and enrolled in this calculation in Figure 4A, 4B and 4C, were severely ill when they diagnosed positive. Since there were a few of the moderate patients who were transformed to severe ones during the treatment, the timeline information of this part of patients is unknown and not enrolled in this calculation.Secondly, among the clustered cases, we tracked $100 \%$ of the clustered infection chains. Due to timely isolation and tracking, most of the clustered cases were in 2-3 clustered infections.

However, some cases have been spread for several generations. Obviously, the clinical features between those different clusters are worth further identification and exploration.

After the COVID-19 outbreak in Wuhan, it rapidly spread almost the world in three stages from sporadic, cluster gathering and the community spreading. Obviously, cluster gather transmission is the intermediate stage and a crucial link in the middle between sporadic to community transmission.

\section{Conclusion}

In conclusion, cutting the transmission chains is timely and effective measures to preventing COVID-19. Early detection of cases and isolation without delay are useful measures to reduce morbidity. These measures not only improve the quality of prevention and control, but also reduce the waste of medical and health resources. Here, experience of Shenzhen City has the practical reference value.

\section{Declarations}

\section{Acknowledgments}

We thank the Health Commission of Shenzhen Municipality for great support and provision of data about diagnosed COVID-19 cases. We acknowledge the love, caring and patience of all of us in confront of SARS-CoV-2 virus.

\section{Funding}

This work was supported by National Natural Science Foundation of China grant number [81804154] and Natural Science Foundation of Shenzhen grant number [JCYJ20190812171807146].

\section{Author information}

Affiliations

Shenzhen University First Affiliated Hospital 3002 Sungang West Road, Shenzhen, Guangdong Province Shenzhen, CN 518000;Shenzhen Institute of Geriatrics, 3002 Sungang West Road, Shenzhen, Guangdong Province 518000, China 
Feijuan Huang

Shenzhen University First Affiliated Hospital 3002 Sungang West Road, Shenzhen, Guangdong Province Shenzhen, CN 518000;Anhui Medical University,81 Meishan Road, Hefei,Anhui Province230032,China

Yu Zhang,Xiaomiao Tian,Shiwei Yang

Shenzhen Technology University, No. 3002, Lantian Road, Pingshan District,

Shenzhen, Guangdong 518118; Shenzhen Wemed Medical Technology Co., Ltd.

No.2092WenjingRoad,Yitai Building,Luohu District, Shenzhen, Guangdong 518003

Yuanzhe Cai

Shenzhen University First Affiliated Hospital 3002 Sungang West Road, Shenzhen, Guangdong Province Shenzhen, CN 518000

Feng Ding, Yan Liu, Yaowei Huang, Zhengzhi Wu

Shenzhen University First Affiliated Hospital 3002 Sungang West Road, Shenzhen, Guangdong Province Shenzhen, CN 518000;Hunan University of Chinese Medicine, Changsha, Hunan, CN

Zhanyan Liu, Jieren Liu

Contributions

FJH,YZ and SWY conceived and designed the study.FD, XMT, ZZL and JL contributed to data collections and data entry; $Y Z C, Y W H$ and $Y L$ contributed to data analysis and picture production.FJH and $Y Z$ drafted the manuscript. ZZW and Jieren Liu provided critical review of the manuscript.All authors reviewed and approved the final version of the manuscript. Thanks to WLL for assistance with the study.

Availability of data and materials

The datasets used and analyzed during the current study are available from the corresponding author on reasonable request by emailing $\mathrm{FJH}$

Ethics approval and consent to participate

This study has been approved by the Ethics Committee of The First Affiliated Hospital of Shenzhen University, Shenzhen.

Consent for publication

Not applicable.

Competing interests 
None declared.

\section{References}

1.Huang C, Wang Y, Li X, et al. Clinical features of patients infected with 2019 novel coronavirus in Wuhan,China.Lancet.2020;395(10223):497-506.Epub2020/01/28.doi:10.1016/s0140-6736(20)30183-5.

2.Gorbalenya AE, Baker SC, Baric RS, et al. Severe acute respiratory syndrome-related coronavirus: The species and its viruses - a statement of the Coronavirus Study Group. bioRxiv. 2020:2020.02.07.937862. doi: 10.1101/2020.02.07.937862.

3.Columbus C, Brust KB, Arroliga AC. 2019 novel coronavirus: an emerging global threat. Proc (Bayl Univ Med Cent). 2020;33(2):209-12.

4.Jin YH, Cai L, Cheng ZS, et al. A rapid advice guideline for the diagnosis and treatment of 2019 novel coronavirus (2019-nCoV) infected pneumonia (standard version). Mil Med Res. 2020;7(1):4. Epub 2020/02/08. doi: 10.1186/s40779-020-0233-6.

5.Song F, Shi N, Shan F,et al. Emerging Coronavirus 2019-nCoV Pneumonia. Radiology. 2020:200274. Epub 2020/02/07. doi: 10.1148/radiol.2020200274.

6.Riou J, Althaus CL. Pattern of early human-to-human transmission of Wuhan 2019 novel coronavirus (2019-nCoV), December 2019 to January 2020. Euro Surveill. 2020;25(4). Epub 2020/02/06. doi: 10.2807/1560-7917.Es.2020.25.4.2000058.

7.Liu T, Hu J, Xiao J, et al. Time-varying transmission dynamics of Novel Coronavirus Pneumonia in China. bioRxiv. 2020:2020.01.25.919787. doi: 10.1101/2020.01.25.919787.

8.Benvenuto D, Giovannetti M, Ciccozzi A, et al. The 2019-new coronavirus epidemic: evidence for virus evolution. bioRxiv. 2020:2020.01.24.915157. doi: 10.1101/2020.01.24.915157.

9. Rothe C, Schunk M, Sothmann P, et al. Transmission of 2019-nCoV Infection from an Asymptomatic Contact in Germany. N Engl J Med. 2020. Epub 2020/02/01. doi: 10.1056/NEJMc2001468.

10.Li X, Wang W, Zhao X, et al. Transmission dynamics and evolutionary history of 2019-nCoV. J Med Virol. 2020. Epub 2020/02/07. doi: 10.1002/jmv.25701.

11.Tuite AR, Fisman DN. Reporting, Epidemic Growth, and Reproduction Numbers for the 2019 Novel Coronavirus (2019-nCoV) Epidemic. Ann Intern Med. 2020. Epub 2020/02/06. doi: 10.7326/m20-0358.

12.Zumla A, Hui DS, Azhar El, et al. Reducing mortality from 2019-nCoV: host-directed therapies should be an option. Lancet. 2020. Epub 2020/02/09. doi: 10.1016/s0140-6736(20)30305-6. 
13.Interim Guildance on New Coronavirus Pneumonia Diagnosis and Treatment (Trial Version 5). Available from:

http://www.chinacdc.cn/jkzt/crb/zl/szkb_11803/jszl_11815/202002/W020200209352220787328.pdf.

14.Zhao S, Musa SS, Lin Q, et al. Estimating the Unreported Number of Novel Coronavirus (2019-nCoV) Cases in China in the First Half of January 2020: A Data-Driven Modelling Analysis of the Early Outbreak. J Clin Med. 2020;9(2). Epub 2020/02/07. doi: 10.3390/jcm9020388.

15.Chen N, Zhou M, Dong X,et al. Epidemiological and clinical characteristics of 99 cases of 2019 novel coronavirus pneumonia in Wuhan, China: a descriptive study. Lancet. 2020;395(10223):507-13. Epub 2020/02/03. doi: 10.1016/s0140-6736(20)30211-7.

16.Ki M, nCo VT. Epidemiologic characteristics of early cases with 2019 novel coronavirus (2019-nCoV) disease in Republic of Korea. Epidemiol Health. 2020:e2020007. Epub 2020/02/10. doi: 10.4178/epih.e2020007.

17.WHO. Report of the WHO-China Joint Mission on Coronavirus Disease 2019 2020. Available from: https://www.who.int/publications-detail/report-of-the-who-china-joint-mission-on-coronavirus-disease2019-(covid-19).

18.Yin Y, Wunderink RG. MERS, SARS and other coronaviruses as causes of pneumonia. Respirology. 2018;23(2):130-7. Epub 2017/10/20. doi: 10.1111/resp.13196.

19.Song Z, Xu Y, Bao L, et al. From SARS to MERS, Thrusting Coronaviruses into the Spotlight. Viruses. 2019;11(1):59. doi: 10.3390/v11010059.

20.Channappanavar R, Fett C, Mack M, et al.Sex-Based Differences in Susceptibility to Severe Acute Respiratory Syndrome Coronavirus Infection. J Immunol. 2017;198(10):4046-53. Epub 2017/04/03. doi: 10.4049/jimmunol.1601896.

21.Badawi A, Ryoo SG. Prevalence of comorbidities in the Middle East respiratory syndrome coronavirus (MERS-CoV): a systematic review and meta-analysis. International journal of infectious diseases. 2016;49:129-33. Epub 2016/06/21. doi: 10.1016/j.ijid.2016.06.015.

22.Jaillon S, Berthenet K, Garlanda C. Sexual Dimorphism in Innate Immunity. Clin Rev Allergy Immunol. 2019;56(3):308-21. Epub 2017/10/01. doi: 10.1007/s12016-017-8648-x.

\section{Figures}



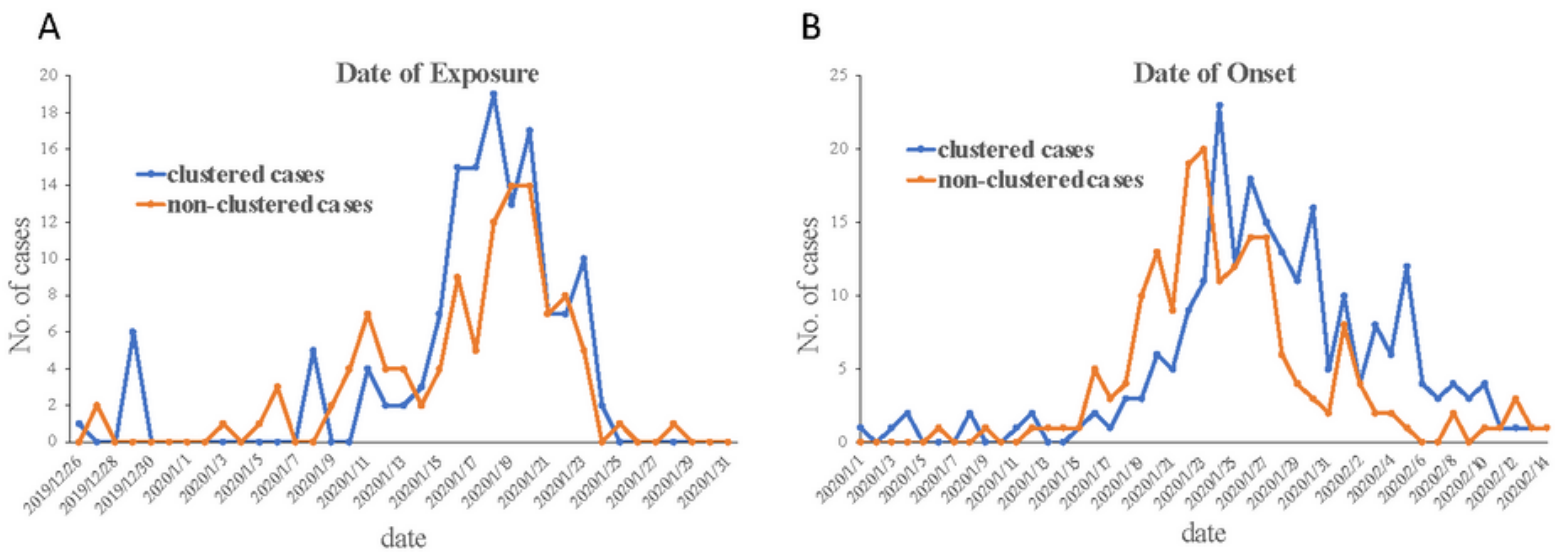

C

D
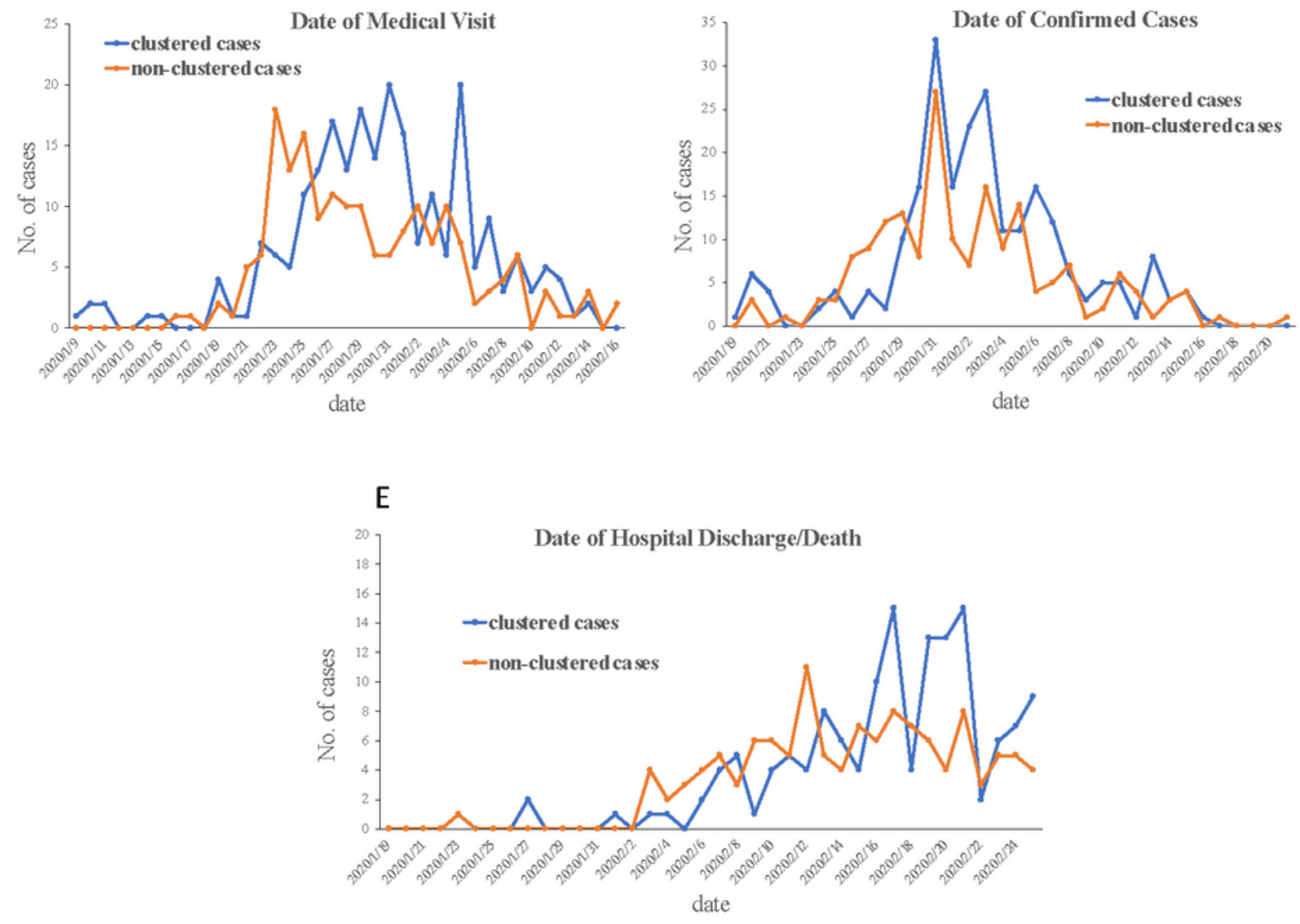

\section{Figure 1}

Comparison between clustered cases and non-clustered cases in Shenzhen (A) Dates of exposure among

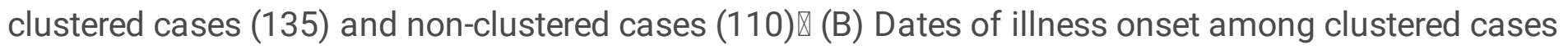

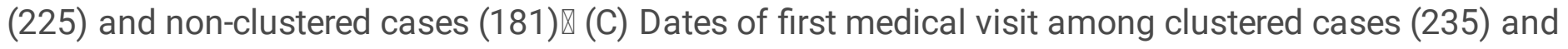
non-clustered cases (182)区 (D) Dates of the cases confirmed among clustered cases (235) and non- 


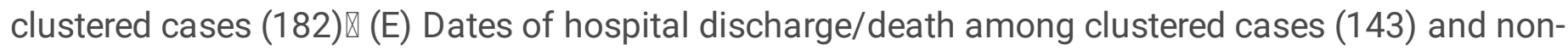
clustered (122) cases.

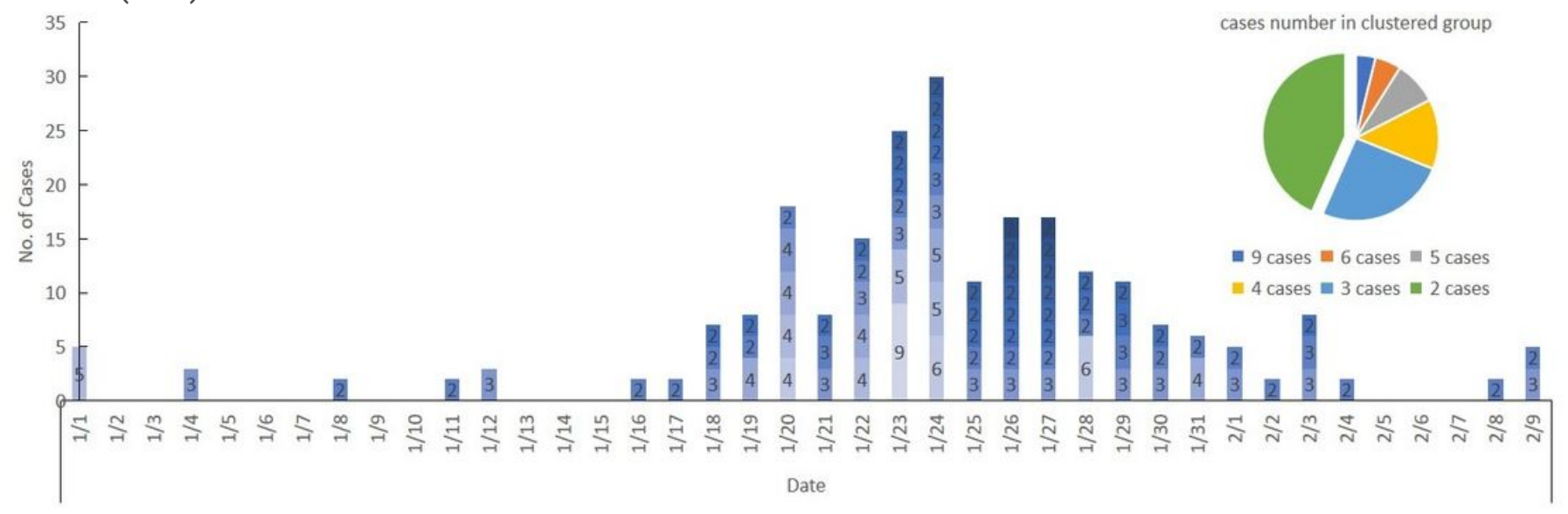

Figure 2

Characteristics of clustered cases in clustered group (A)Dates of illness onset in each clustered group (B) The diagram of the typical transmission path of clustered cases 


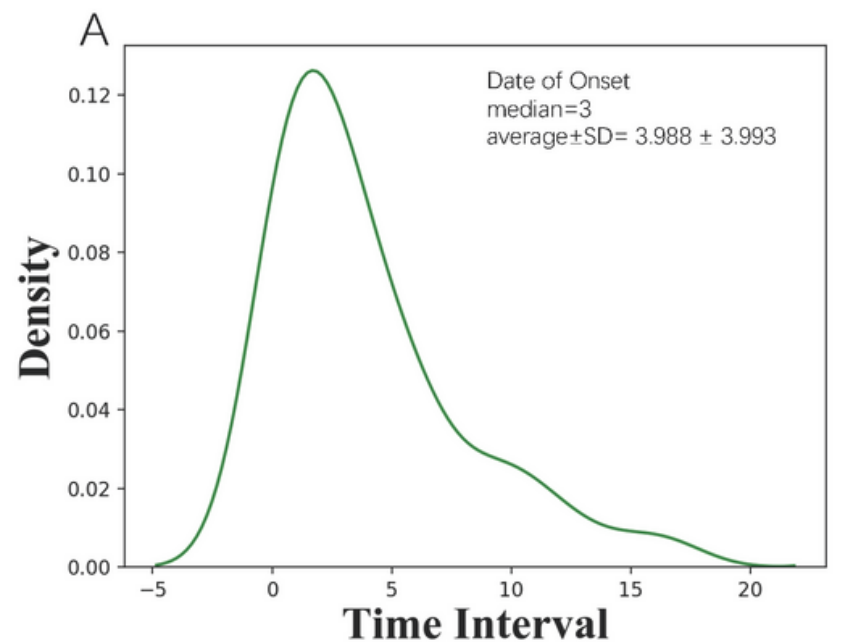

B
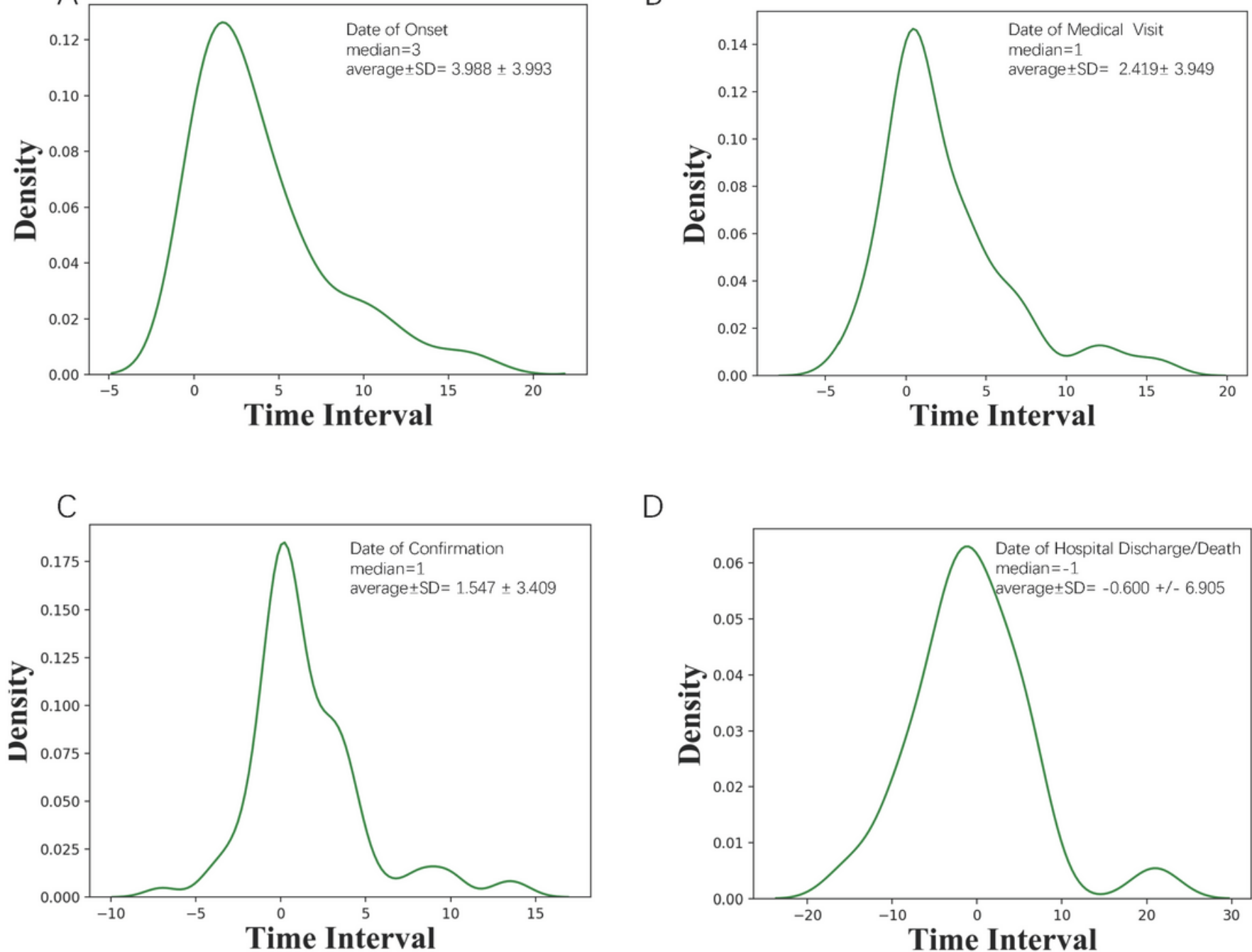

D

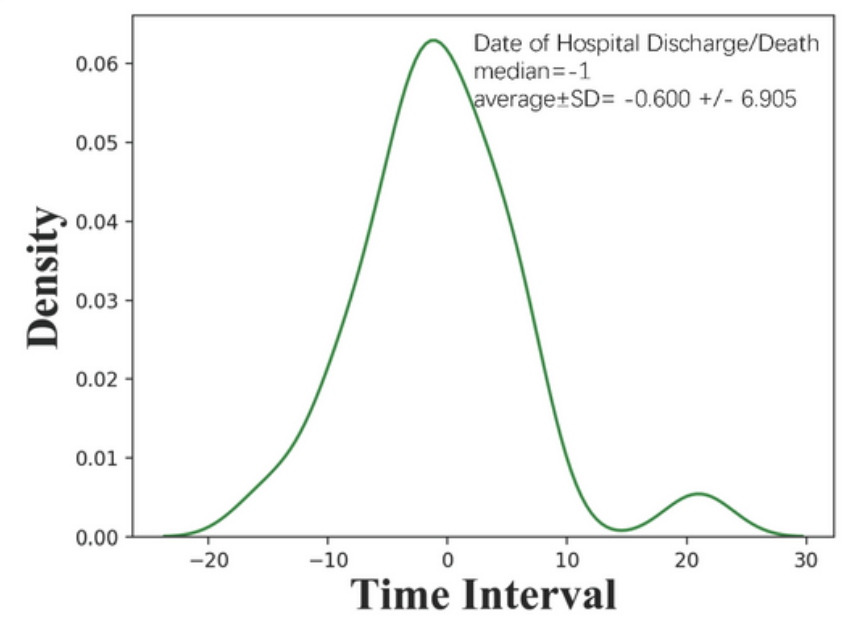

Figure 3

Comparison between the first case and the second case in the clustered group (A) Time intervals of illness onset between the first case and the second infection case in each 86 clustered groups ; (B) Time intervals of first medical visit between the first case and the second case in each 86 clusters; (C) Time intervals of confirmation between the first case and the second case in each 86 clusters; (D) Time intervals of hospital discharge/death between the first case and the secondary case in the 26 clusters. 

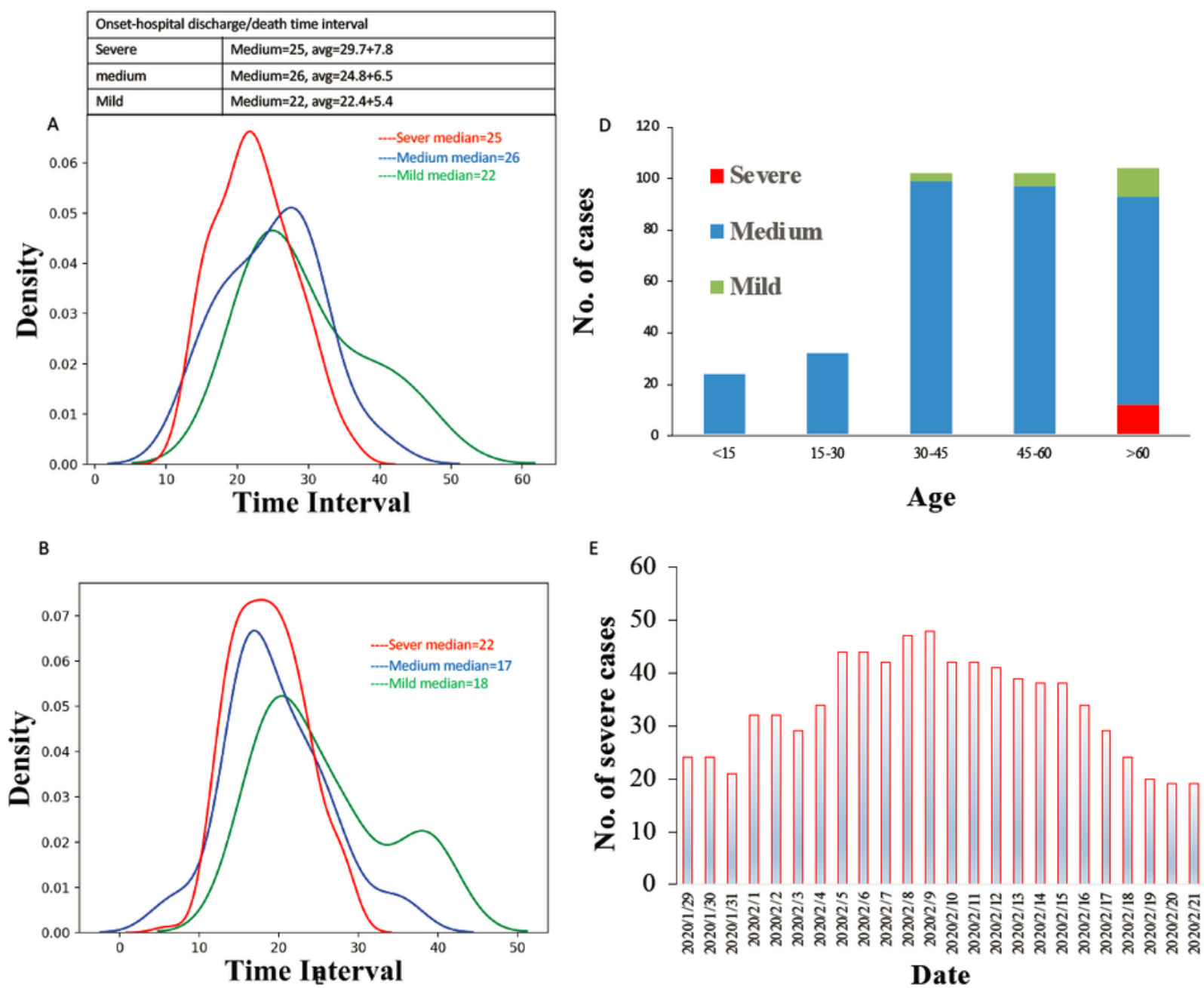

\section{Date}
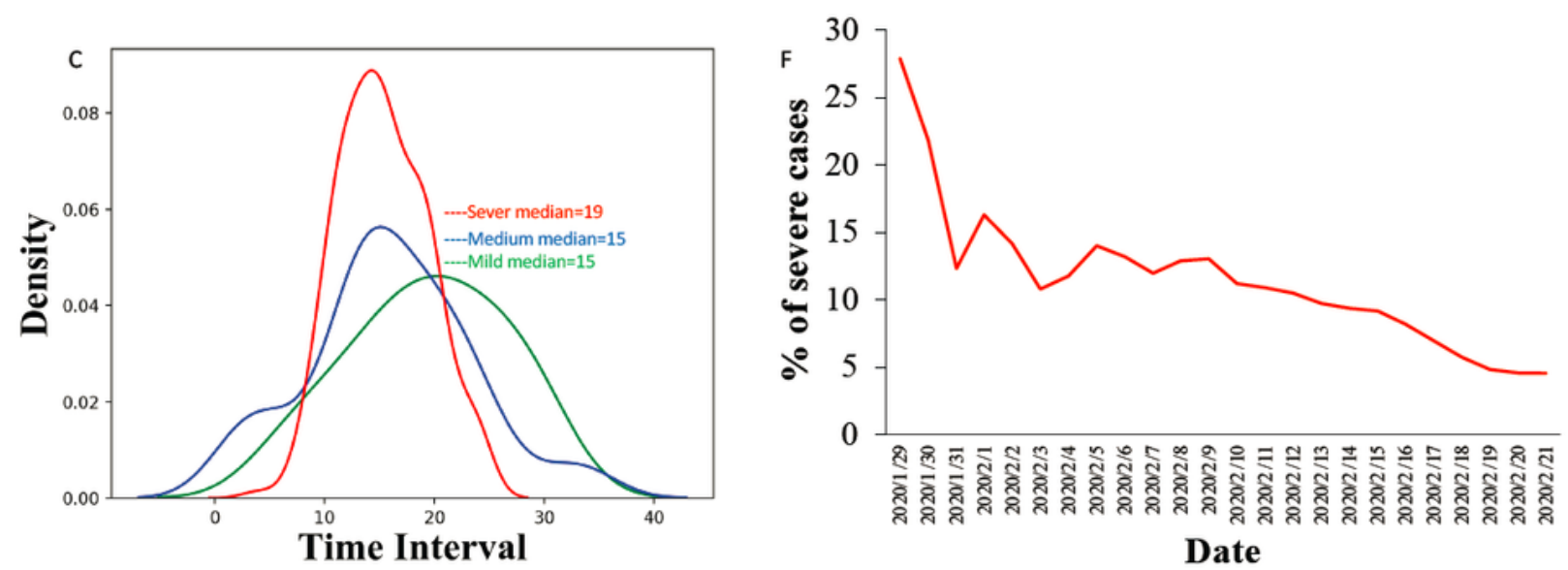

\section{Figure 4}

Severity and time course of the COVID-19 patients in Shenzhen (A) Time intervals between the illness onset and hospital discharge/death in mild, medium , and severe groups; (B) Time intervals between the medical visit and hospital discharge/death in mild, medium, and severe groups; (C) Time intervals between the case confirmation date and hospital discharge/death date in mild, medium and severe 
groups; (D) Age distribution of the mild, medium and severe patients; (E) Dates of the number of severe patients; (F) Dates of the percentage of severe patients.
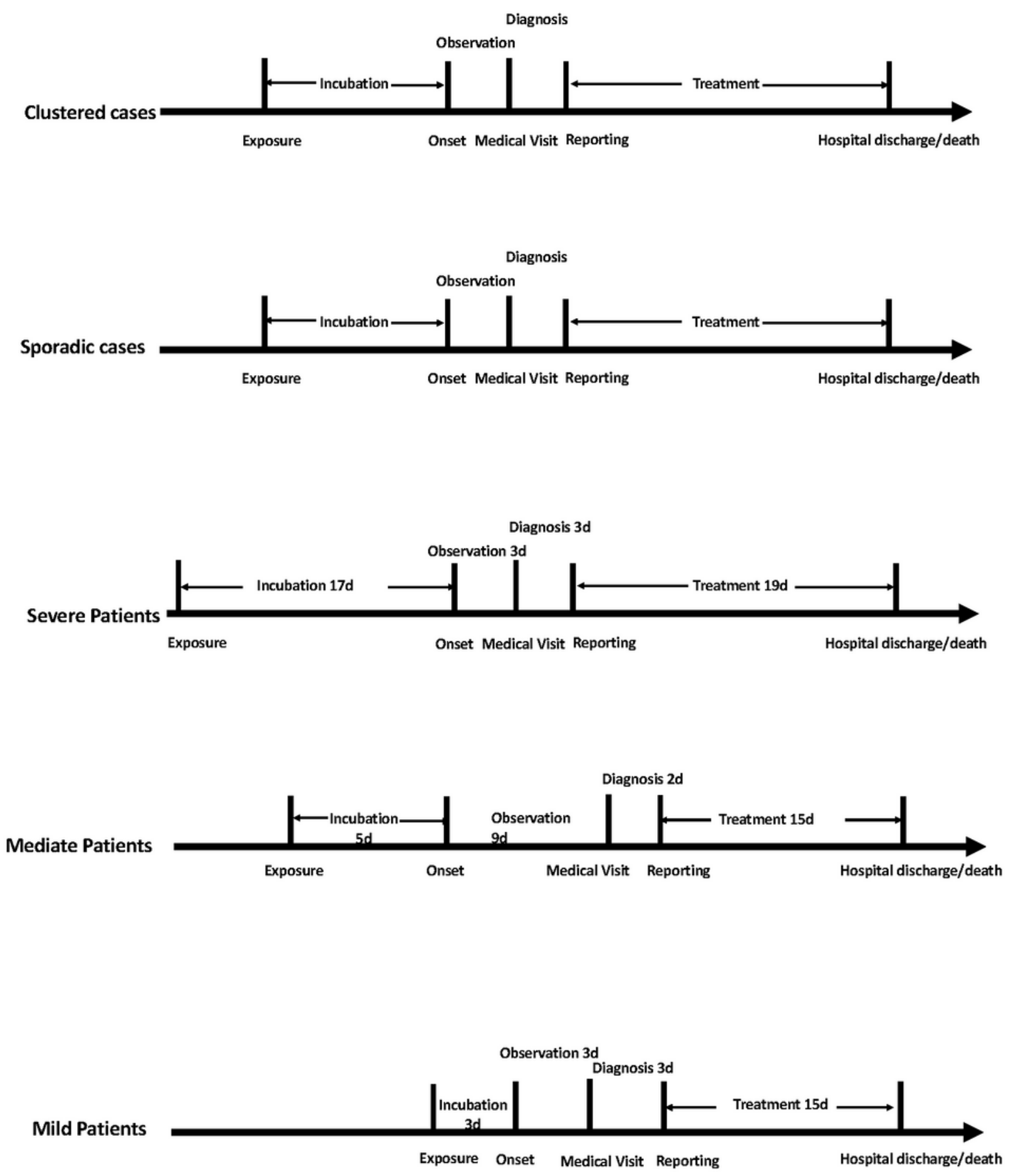

\section{Figure 5}

Epidemical and pathological time courses 